Thorax (1972), 27, 33.

\title{
Pulmonary aspergillosis caused by Aspergillus flavus
}

\author{
E. S. MAHGOUB and A. M. EL HASSAN \\ Departments of Microbiology and Pathology, Faculty of Medicine, University of Khartoum
}

Bronchopulmonary aspergillosis is usually caused by Aspergillus fumigatus. The six cases from the Sudan reported here were due to Aspergillus flavus. Three of the patients had primary invasive disease, one had Aspergillus mycetoma in a pre-existing lung cavity, and two had allergic aspergillosis. A special feature of the invasive disease was the clinical and radiographic simulation of malignancy, particularly in one patient who had multiple disseminated nodules of fungal granulomata in the skin of the lower abdomen.

Fungi of the genus Aspergillus are filamentous organisms that produce airborne spores and have a wide distribution as saprophytes in nature. Occasionally they become pathogenic to man and cause a variety of lesions, the most important of which is pulmonary disease. The latter appears in well-recognized clinical forms for which several classifications have been suggested (Gowing and Hamlin, 1960; Campbell and Clayton, 1964; Kennedy, Malone, and Blyth, 1970). Another type of aspergillosis is that affecting the paranasal sinuses, a rare condition elsewhere but not uncommon in the Sudan (Sandison, Gentles, Davidson, and Milošev, 1967; Milošev, Mahgoub, Abdel A'al, and El Hassan, 1969).

This paper describes the clinical types of pulmonary aspergillosis in the Sudan where, like the paranasal form, it is caused by Aspergillus flavus and not $A$. fumigatus, which is the commoner pathogen in many other parts of the world.

T A B LE

PULMONARY ASPERGILLOSIS: CLINICAL, RADIOGRAPHIC AND LABORATORY DATA

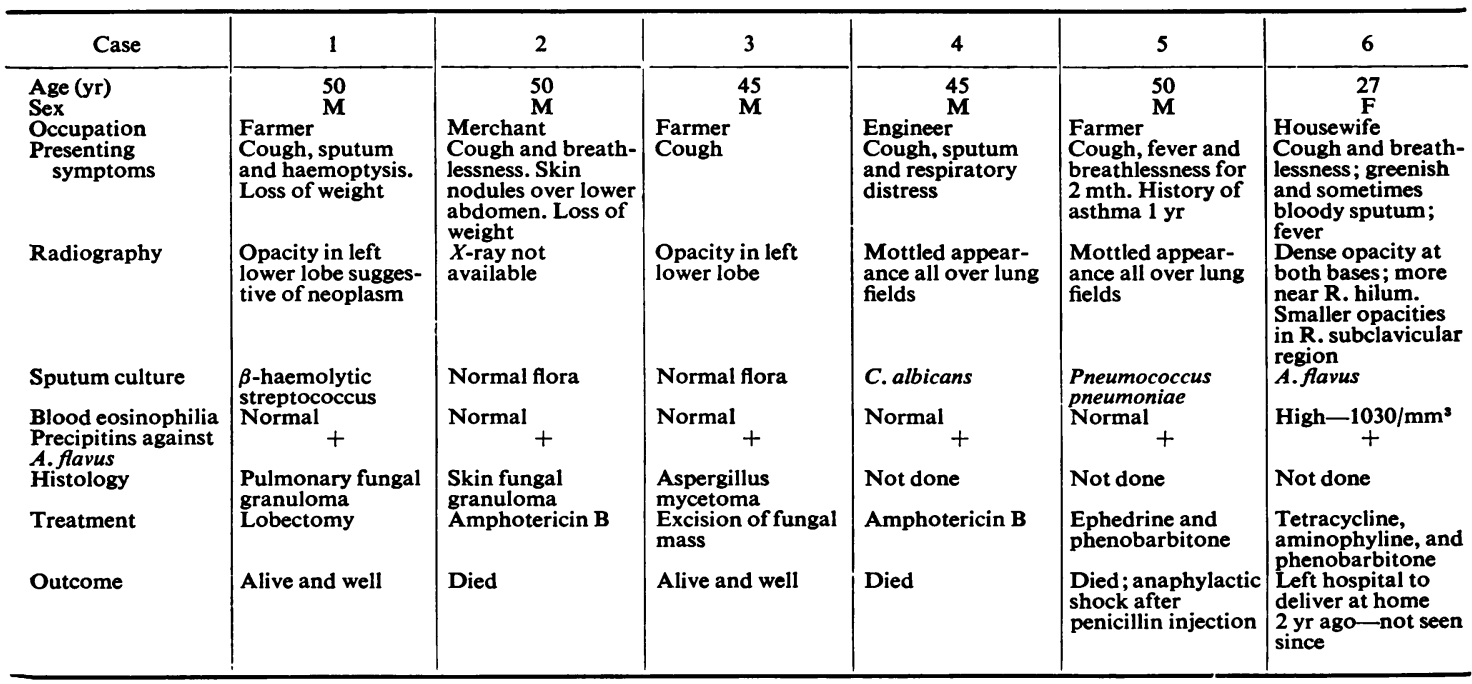




\section{METHODS AND MATERIALS}

This study includes six patients who were seen at the Sha'ab Chest Hospital in Khartoum between 1968 and 1971. The available clinical, radiographic, and laboratory data are shown in the Table. Sputum specimens were stained by the Ziehl-Neelsen method and cultured for bacteria and fungi daily for seven days. Blood agar, Lowenstein Jensen medium, and Sabouraud's dextrose agar with chloramphenicol were used. Total, differential white cell, and eosinophil counts were done. The serum from each patient was tested by means of Ouchterlony agar-gel diffusion for precipitins against four strains of $A$. flavus, $A$. fumigatus, $A$. terreus, $A$. nidulans, and $A$. niger. Antigens from these fungi were prepared by ultrasonic disruption of a float culture in phosphate-buffered saline and precipitation with acetone (Mahgoub, 1971). Pathological studies were performed on the left lower lobe from case 1, a subcutaneous nodule from case 2, and a resected pulmonary tumour from case 3 . Paraffin sections of formalin-fixed tissue were stained with haematoxylin and eosin (H.E.), periodic acid Schiff (PAS), and methenamine silver. No culture was made from these tissues because all specimens were submitted in formalin.

\section{RESULTS}

The Table shows a summary of the clinical, radiographic, and laboratory data. All the patients but

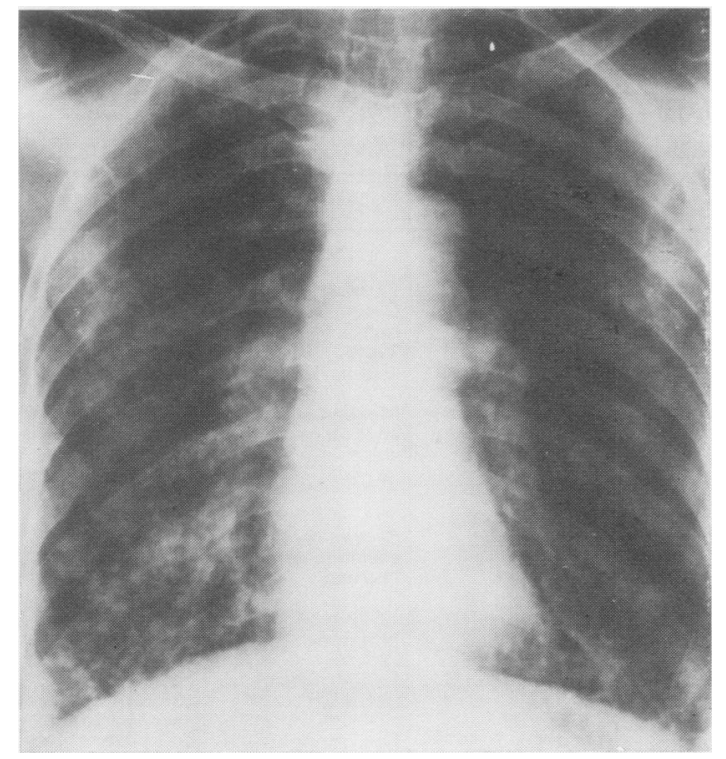

FIG. 1. Case 5. Chest radiograph showing generalized mottled appearance.

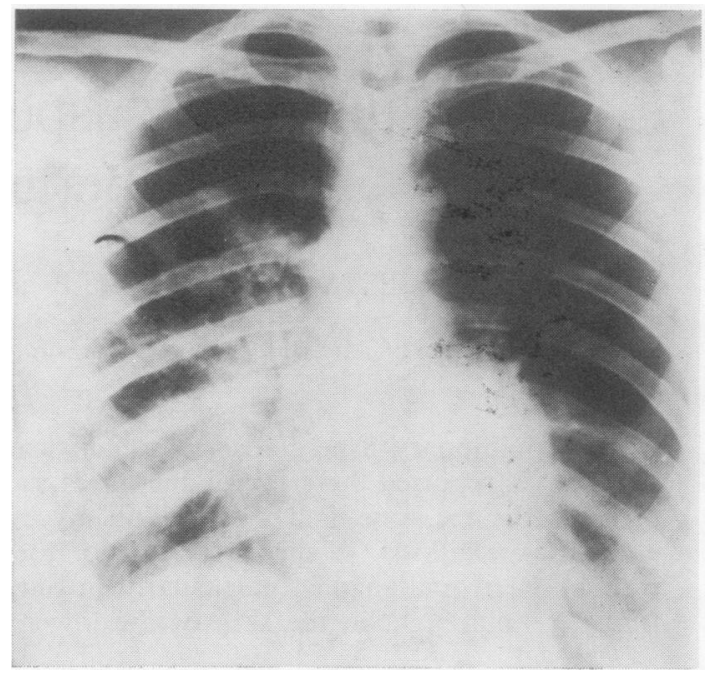

FIG. 2. Case 6. Chest radiograph showing dense opacities at both lung bases and hila, especially on the right side.

one were males whose ages were either 45 or 50 years. The single female was 27 years old. Three of the patients were farmers and patient 2 had been a farmer earlier in life. The major symptoms were cough and breathlessness. Haemoptysis occurred in only one patient. Radiographic examination showed either generalized mottling (Fig. 1) or opacities mainly at the lung bases (Fig. 2). Apart from their fungal infection these patients were healthy adults with the exception of patient 3 , who had a pre-existing lung cavity. In particular, no patient had evidence of paranasal aspergillosis and none was on steroid or antibiotic therapy. The diagnosis of aspergillosis was established by demonstration of precipitins against $A$. flavus antigens in all cases, coupled with the presence of fungal hyphae that looked like Aspergillus species in histological sections from cases 1,2, and 3; and a positive culture of $A$. flavus from the sputum in case 6 .

SEROLOGY Precipitation arcs developed against $A$. flavus in all cases. No precipitation lines were seen with $A$. fumigatus, $A$. terreus, $A$. nidulans, or $A$. niger. These positive lines were sharp and more than one line developed with all sera, as Fig. 3 from case 2 shows.

CULTURE Sputum from case 1 yielded a $\beta$-haemolytic streptococcus and that from case 4 Candida albicans. From case 5 Pneumococcus pneumoniae was isolated and A. flavus was grown from case 6 . 


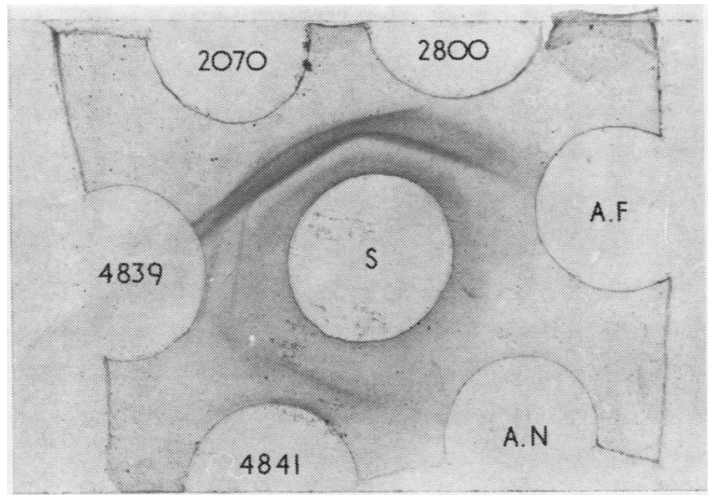

FIG. 3. Case 2. Gel diffusion plate of serum against Aspergillus antigens:

\begin{tabular}{|c|c|}
\hline $\left.\begin{array}{l}2070 \\
2800 \\
4839 \\
4841\end{array}\right\}$ & A. flavus strains \\
\hline $\begin{array}{l}A . F . \\
A . N . \\
S\end{array}$ & $\begin{array}{l}\text { A. fumigatus } \\
\text { A. nidulans } \\
\text { Patient's serum. }\end{array}$ \\
\hline
\end{tabular}

\section{PATHOLOGY}

CASE 1 (Fig. 4) Arising in the left lower lobe bronchus was an irregular mass of yellowish white tissue measuring $2.7 \mathrm{~cm}$ in its greatest diameter. It

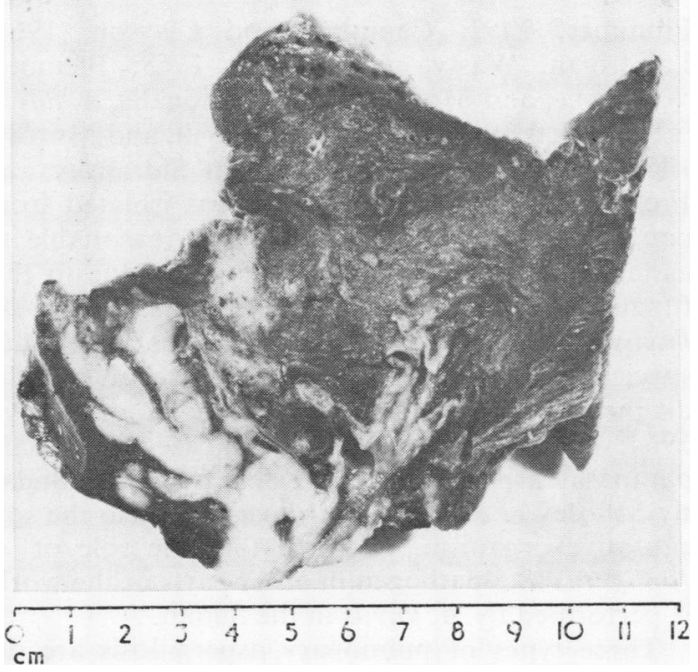

FIG. 4. The cut surface of the left lower lobe showing a tumour-like lesion arising in the wall of the lower lobe bronchus. Note bronchiectasis of branches to the apical segment.

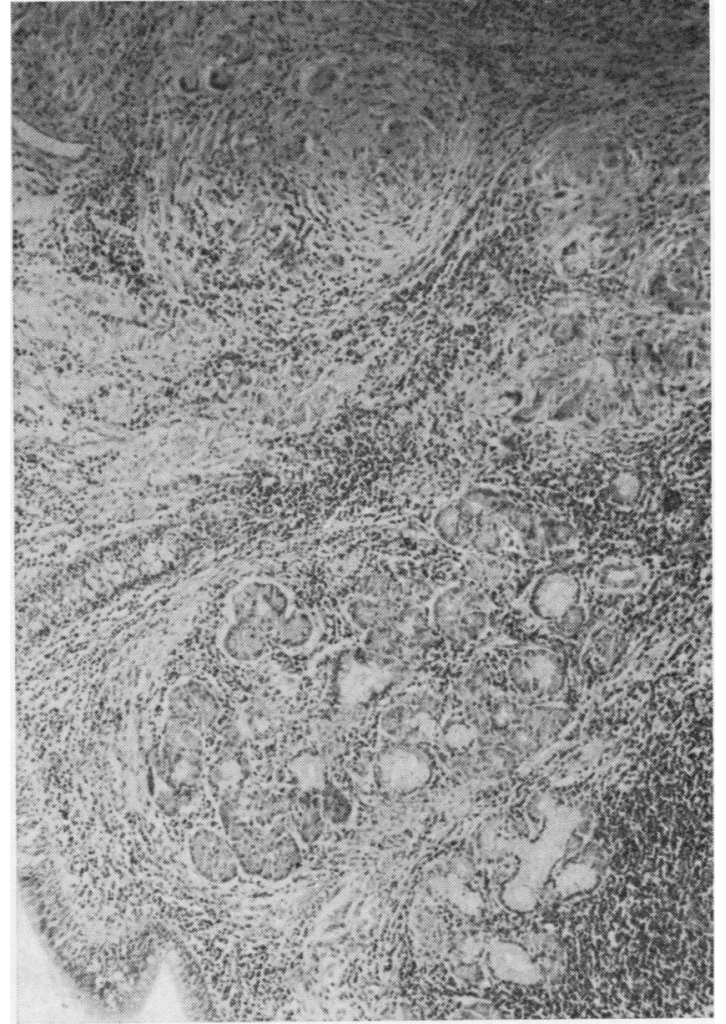

FIG. 5. Section of the lower lobe bronchus showing multiple granulomata adjacent to the bronchial gland. H.E. $\times 73$.

was firm in consistency and the cut surface was of a homogenous close texture. The lumen of the main lower lobe bronchus was slightly narrowed and the overlying mucosa was apparently intact. The bronchial branches to the apical portion of the lobe were surrounded and stenosed by the mass at their origin, dilated distally, and they contained inspissated mucus. The rest of the lung showed patches of early bronchopneumonia. Histology of the mass showed several granulomata scattered in a dense hyaline fibrous tissue stroma. These granulomata were composed of epithelioid and multinucleated giant cells and in the centre of some there were small microabscesses (Fig. 5). The PAS and silver stains showed septate fungal hyphae which occasionally branched laterally, inside the giant cells, and in the microabscesses (Fig. 6). Focal areas of fibrinoid necrosis but without related fungal elements and with peripheral palisading of histiocytes were also seen. Some arteries showed an inflammatory reaction involving all coats composed 


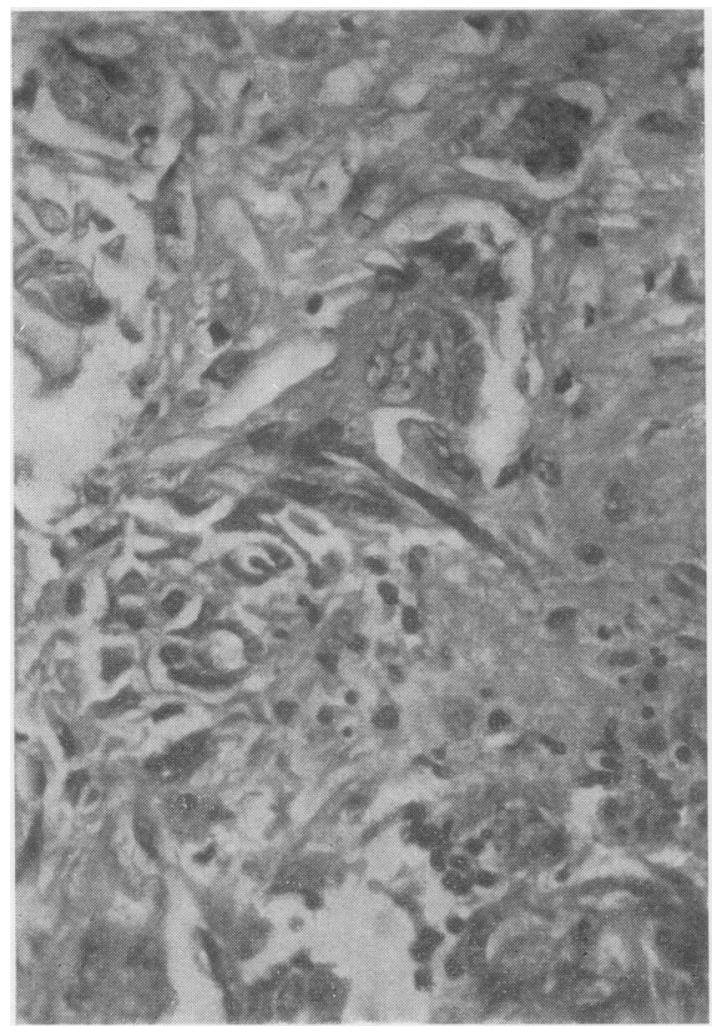

FIG. 6. Fungal granuloma showing a septate hypha inside a multinucleated giant cell. $P A S \times 365$.

of lymphocytes, histiocytes, and neutrophils but fungal invasion of the vessel wail was not observed (Fig. 7). The inflammatory granulomatous reaction invaded the bronchial cartilage and there were small mucosal ulcers.

CASE 2 The specimen received was an irregular mass of subcutaneous tissue measuring $1 \mathrm{~cm}$ in its greatest diameter. It was unencapsulated and firm in consistency and the cut surface was yellowish white and homogenous. Histopathology showed the same features as in case 1 with the reaction involving the dermis and subcutaneous tissues.

CASE 3 The surgically removed specimen measured $4 \mathrm{~cm}$ in its greatest diameter. It was of a light brown colour and friable. Histopathology showed tangled fungal hyphae with septa and branching. Some of these carried vesicles typical of Aspergillus species.

\section{DISCUSSION}

The commonest cause of bronchopulmonary aspergillosis is $A$. fumigatus (Hinson, Moon, and

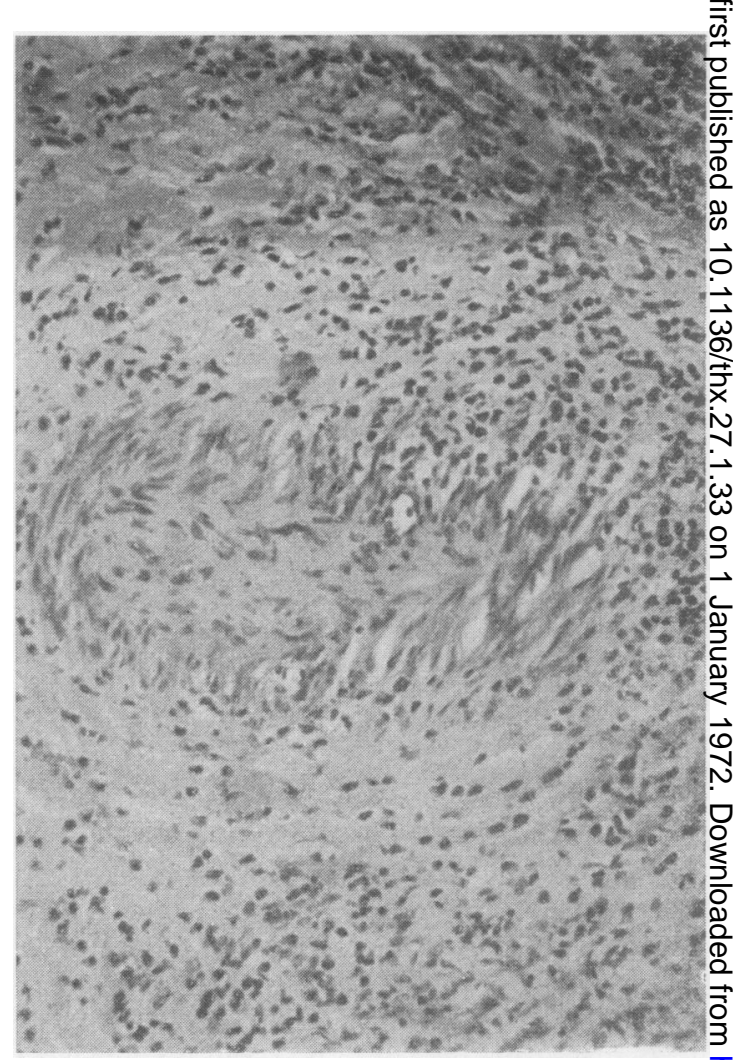

FIG. 7. A tangentially cut artery, the wall of which show acute and chronic inflammatory reaction. H.E. $\times 180$.

Plummer, 1952; Campbell and Clayton, 1964, Landrigan, Wasty, and Nigam, 1968; Ikemoto $\stackrel{0}{x}$ Watanabe, and Mori, 1971). Occasionally A. flavus is the causal organism (Finegold, Will, and Murray 1959; Naji, 1959; Carbone, Sabesin, Sidransky, and Frei, 1964). Although $A$. flavus was isolated from one patient only in our series it is reasonable tog assume that in all cases infection was due to this fungus as sera from all patients reacted with $A$ 을 flavus antigens and not with any other Aspergillusu species. The precipitin test is fairly reliable in the diagnosis of aspergillosis (Longbottom and Pepys 1964; Walter and Jones, 1968). In cases of primar paranasal aspergilloma reported from the Sudaw by Milošev et al. (1969) A. flavus was also the sole causative organism. It seems that the role of $A_{\mathbb{\Phi}}^{\complement}$ fumigatus as a pathogen in other parts of the worlọ is performed by $\boldsymbol{A}$. flavus in the Sudan.

Three types of pulmonary aspergillosis are de monstrated in this series. Cases 1,2 , and 4 are of primary and invasive nature. There was no othe $\mathbb{B}$ associated disease. Isolation of $C$. albicans from the sputum of case 4 is of no significance because the 
precipitation test against $C$. albicans was negative and the agglutination test was of very low titre. Case 3 was a typical aspergillus mycetoma filling a cavity which was probably tuberculous in nature. Cases 5 and 6 were examples of the allergic type of aspergillosis. Patient 5 had a history of asthma and patient 6 had wheezing sounds over the lungs and a very high eosinophil count.

The clinical features of patients 1 and 2 suggested a carcinoma of the bronchus; the patients presented with cough, haemoptysis, and loss of weight, and in addition patient 2 had multiple skin nodules simulating metastases. Disseminated aspergillosis, with nodules in the skin, was reported previously by Vedder and Schorr (1969).

It is almost certain that the bronchiectasis in case 1 was the effect rather than the cause of the fungal granuloma; the patient had no history of antecedent respiratory disease and the healthy looking but dilated bronchi did not show evidence of bacterial or fungal infection. We believe that the histopathological changes in the first two cases are due to two factors: first, the invasive nature of the fungus and, second, the associated immunological reaction which is suggested by the presence of fibrinoid necrosis and arteritis. The role of fungal metabolites or toxin cannot be excluded.

We should like to acknowledge the help of Dr. A'bdin Sharaf, Dr. Sir Abdel Magid, and Mr. Ahmed Abdel Aziz of the Chest Hospital. Our thanks are also extended to Dr. Habib Abdalla, who reported the chest radiographs, and to Miss Christine Philpot, of the Mycological Reference Laboratory, London School of Hygiene and Tropical Medicine for typing the manuscript.

\section{REFERENCES}

Campbell, M. J., and Clayton, Y. M. (1964). Bronchopulmonary aspergillosis. Amer. Rev. resp. Dis., 89, 186.
Carbone, P. P., Sabesin, S. M., Sidransky, H., and Frei, E. (1964). Secondary aspergillosis. Ann. intern. Med., 60, 556.

Finegold, S. M., Will, D., and Murray, J. F. (1959). Aspergillosis; a review and report of 12 cases. Amer.J. Med., 27, 463.

Gowing, N. F. C., and Hamlin, I. M. E. (1960). Tissue reactions to Aspergillus in cases of Hodgkin's disease and leukaemia. J. clin. Path., 13, 396.

Hinson, K. F. W., Moon, A. J., and Plummer, N. S. (1952). Broncho-pulmonary aspergillosis; a review and a report of eight new cases. Thorax, 7, 317.

Ikemoto, H., Watanabe, K., and Mori, T. (1971). Pulmonary aspergilloma. Sabouraudia, 9, 30.

Kennedy, W. P. U., Malone, D. N., and Blyth, W. (1970). Necrotizing pulmonary aspergillosis. Thorax, 25, 691 .

Landrigan, P. L., Wasty, G., and Nigam, S. (1968). Pulmonary aspergillosis: report of seven cases. Canad. med. Ass. J., 98, 642.

Longbottom, J. L., and Pepys, J. (1964). Pulmonary aspergillosis: diagnostic and immunological significance of antigens and C-substance in Aspergillus fumigatus. J. Path. Bact., 88, 141

Mahgoub, E. S. (1971). Mycological and serological studies on Aspergillus flavus isolated from paranasal aspergilloma in Sudan. J. trop. Med. Hyg., 74, 162.

Milošev, B., Mahgoub, E. S., Abdel A'al, O., and El Hassan, A. M. (1969). Primary aspergilloma of paranasal sinuses in the Sudan. Brit. J. Surg., 56, 132.

Naji, A. F. (1959). Bronchopulmonary aspergillosis; report of two new cases, review of literature, and suggestion for classification. Arch. Path., 68, 282.

Sandison, A. T., Gentles, J. C., Davidson, C. M., and Milošev, B. (1967). Aspergilloma of paranasal sinuses and orbit in Northern Sudanese. Sabouraudia, 6, 57

Vedder, J. S., and Schorr, W. F. (1969). Primary disseminated pulmonary aspergillosis with metastatic skin nodules. J. Amer. med. Ass., 209, 1191.

Walter, J. E., and Jones, R. D. (1968). Serologic tests in diagnosis of aspergillosis. Dis. Chest, 53, 729. 